\title{
Controlling Robot through SMS with Acknowledging facility
}

\author{
Mr. Vipin Dhanraj Vaidya, Dr. Sanjay L. Haridas
}

\begin{abstract}
Robotics plays an integral role in all aspects of manufacturing, medicine, industries, home basic and more and it will take on even greater importance in the future. Controlling Robot through SMS discussed a kind of design of robot based on ARM. All the motions of the robot are controlled by the SMS. The ARM7is used as a controlling unit combining with the GSM TC35i modules. Using GSM networks, a control system has been proposed that will act as an embedded system which can monitor and control appliances and other devices locally using built-in input and output peripherals through the long distances. So we can eliminate the need of being physically present in any location for tasks involving operation of appliances.
\end{abstract}

Keywords: Driver IC and DC motor, GSM modem, LPC2148 (ARM7), PIR sensor

\section{Introduction}

Conventionally in a voice controlled robot, voice is a command received by the voice recognizing kit or the software. The performance of the system is affected by the parameters like speech level, speech matching, surrounding noise etc. As these problems are unavoidable they are directly affects the final result.

Wireless-controlled Robots use RF circuits, which have limited working range, limited frequency range and limited control.

Use of a mobile phone for robotic control can overcome these limitations. It provides the advantage of robust control, working range as large as the area of the service provider, no interference with other controllers. Although the appearance and capabilities of robot vary vastly, all robots share feature of a mechanical, movable structure under some form of control. The control of Robot involves three distinct phases: perception, processing and action. Generally, the perception is done by receiving the SMS from the users mobile which is act as movement command. Processing is done by the on-board microprocessor, and the task is performed using motors or with some other actuators. In this project the robot is connected to GSM modem which is controlled by user mobile phone. With the help of AT commands we can move our robot in desired direction as per our requirement. After completion of the task the robot will send the positive or negative acknowledgement that knowing the user about completion or incompletion of task respectively.

\section{Components Of The System}

There are two components of the system

a. Software

b. Hardware

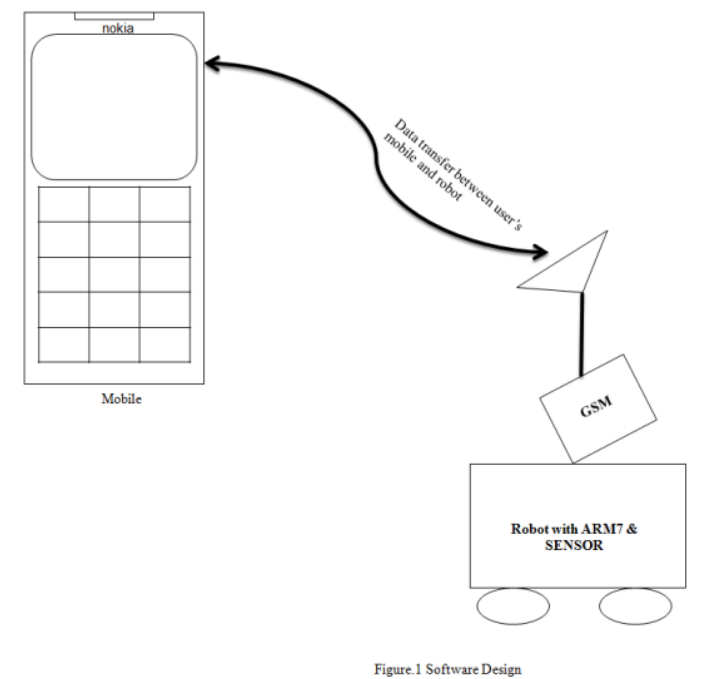

The software part of the system mainly consist two part of programming,

a. Programming part for the GSM is continuously scanning the memory of the SIM mounting the GSM module to send and receive the data (acknowledgement / command) from the robot. 
b. Program for the LPC 2148 microprocessor to control the robot, send instruction to the driver and receives the data from the sensor and send data to the mobile user.

The hardware part is consist of two components a. Mobile: It is unit from which the user sends the commands in the form of SMS to control the motions of the robot.

b. Robot: the robot is consisting of the arm7 as a controlling system, GSM for the receiving and sending the SMS. It also has the driver to drive DC motor, power supply.

\section{Hardware}

Hardware part of the system is mainly consisting of the microprocessor LPC 2148 which is the heart of the system. It also has the DC motor driver to drive the robot according to user's the command. The obstacle sensor is used detect obstacle when the robot in moving condition. So when it detects the obstacle the SMS is send to the user.

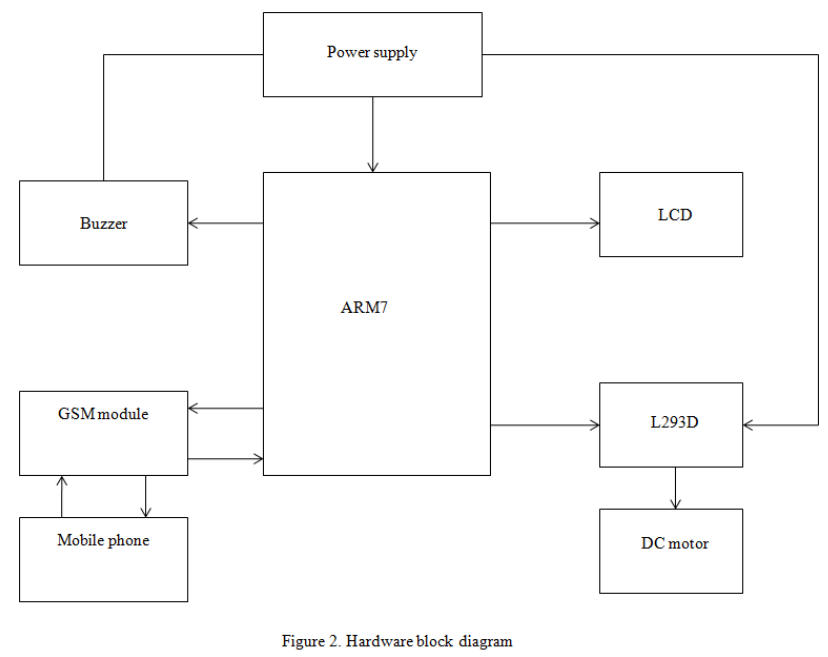

The hardware part also consists of the power supply. Mainly the ARM controller needs 3.3 volt power supply. To use these parts we need to build a regulated 3.3 volt source. Here we use a LM317 voltage regulator IC. LM317 is the standard an integrated three-terminal adjustable linear voltage regulator. LM317 is a positive voltage regulator supporting input voltage of $3 \mathrm{~V}$ to $40 \mathrm{~V}$ and output voltage between $1.25 \mathrm{~V}$ and $37 \mathrm{~V}$. A typical current rating is $1.5 \mathrm{~A}$ although several lower and higher current models are available.

\section{A. Controlling Unit}

LPC2148 is one of the families of microprocessor from ARM7 TDMI. This controller has greatest advantage compared to remaining families of controllers available like PIC and AT mega, those are on chip resources availability (two UART's, two fast I2C-bus, SPI etc) and speed of operation (operating frequency is 0 to $100 \mathrm{MHz}$ ). This LPC2148 has 44 fast GPIO lines, hence we can interface many I/O devices and it is a 64pin controller.

The LPC2148 microcontrollers are a 32-bit ARM7TDMI-S CPU with real-time emulation. It embedded high-speed flash memory ranging from $32 \mathrm{kB}$ to $512 \mathrm{kB}$. It has 16-bit Thumb mode reduces code by more than 30 $\%$ with minimal performance penalty.

The interfacing of the lpc2148 with LCD, GSM is shown in the below, 


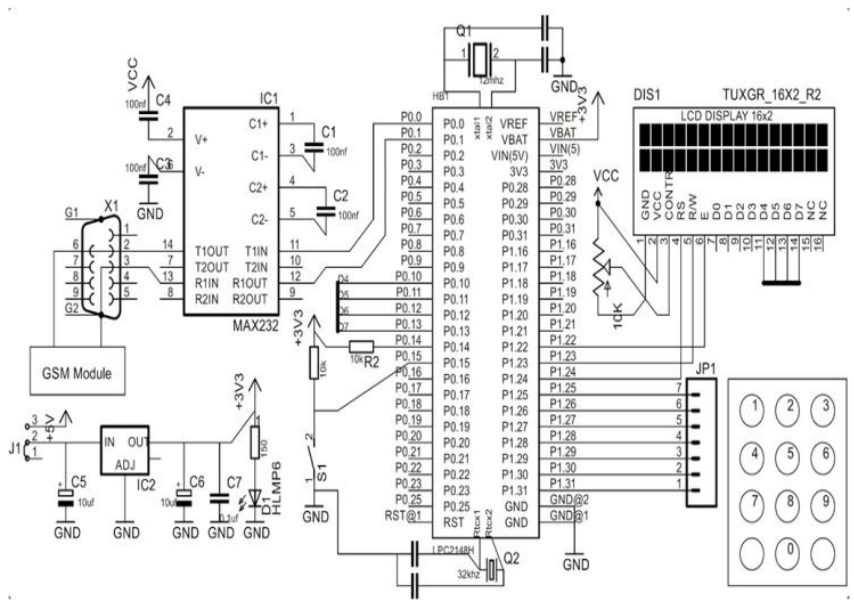

Figure 3. Schematic for Ipc2148 intefface with LCD and GSM

\section{B. Motor Driving circuit}

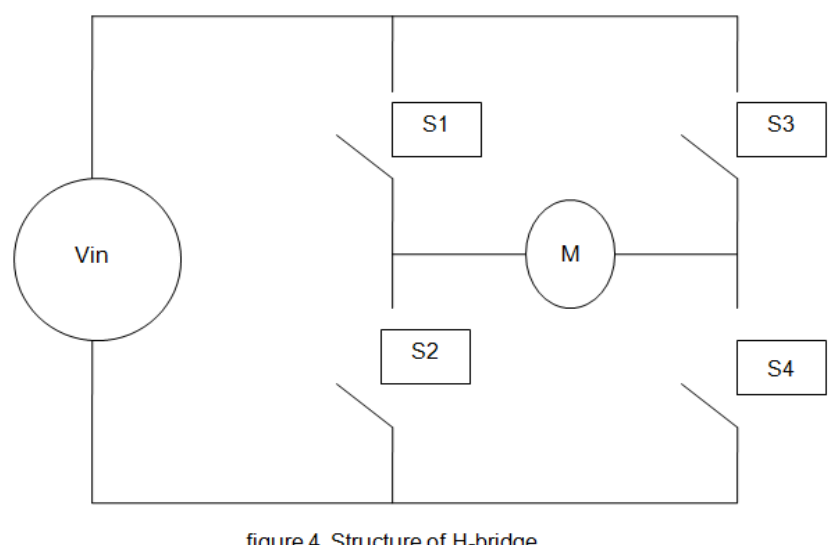

L293D is a motor driving IC used to drive the motors attached to robot. L293D is a transistor H-bridge circuit used to drive the at most two motors at a time. H-bridge means it contains four switches which controls the direction of motor.

This L293D takes PWM signal from controller in order to drive the motor. The speed of motor depends on the PWM signal on \& off pulses. The motor stops rotating during off pulse and runs during on pulse. If the width of the pulse is high motor rotates fast and vice versa.

L293D IC is 16pin DIP package, each motor having separate enable pins. The PWM train pulses applied to the motor.

But hardware is the common thing in the robot; this paper is concentrates on the controlling method and also on the acknowledging method through the SMS using mobile phones.

\section{Communication Between Mobile And Robot}

In the paper GSM is used as a communication media. For the communication of user mobile to the GSM contained robot, one first should know both robot and user should be in service network. The GSM on the robot is connected to the LPC 2149 with the help of RS-232.

Since the short text messaging is popularly known as the SMS is most cheaply and commonly and widely available in mobile service. It is used to control our robot. The SMS as specified by the Etsi organization can be up to 160 character long where each character is7 bits according to the 7- bit default alphabet, eight - bit message are usually not viewable by the phones as text message; instead they are used for data.

\section{Algorithm For Robot Control}

Before running the main program, LPC2148 controller must initialize the runtime environment, which is, writing start code for ARM chips, including the exception vector table, stack initialization, the storage system initialization and target board initialization, usually written in assembly language. If you use the controlling the robot on the basis of GSM while using the SMS service, the program flow chart is shown in Fig.5 


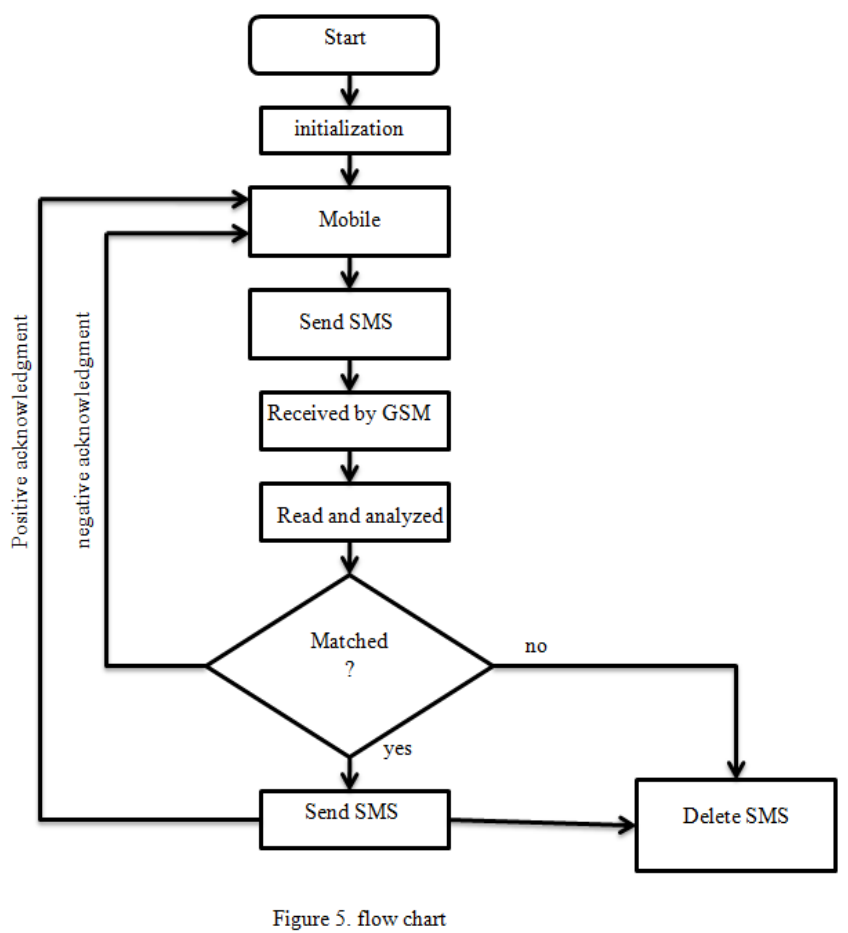

The key of software design is to write function Functions. Functions are realized by calling the corresponding function, which includes two parts: sending and receiving text messages, matching and executing the command. The message is first generated by the user and sends to the robot. If any message is received by the GSM then microcontroller will do next step otherwise it will wait for message. After receiving the message, it will read and analyzed by the microcontroller. If it is error free command then it will run that commands and process according to that. After processing on the message microcontroller generate the positive acknowledgement that shows the completion of task. Otherwise if there is any error in command then negative acknowledgement will generate by the microcontroller and send to user mobile.

\section{Acknowledgment Facility}

After performing the specific task the microcontroller generates the acknowledging message for the user. It sends by the microcontroller using GSM modem. The acknowledge is positive or negative depending upon the completion of the work by the robot.

The PIR sensor used to detect obstacle come in front while robot doing its work. If it happened then microprocessor generates a message and sends to user. User sends the direction changing command to robot to change the direction.

\section{Result Analysis}

The paper on "Controlling Robot through SMS with Acknowledging facility" at the title suggests is aimed to construct a control system that enables the complete control of the interface on which it is based. The fig. 6 shows an implemented snap of the Controlling Robot through SMS with Acknowledging facility.

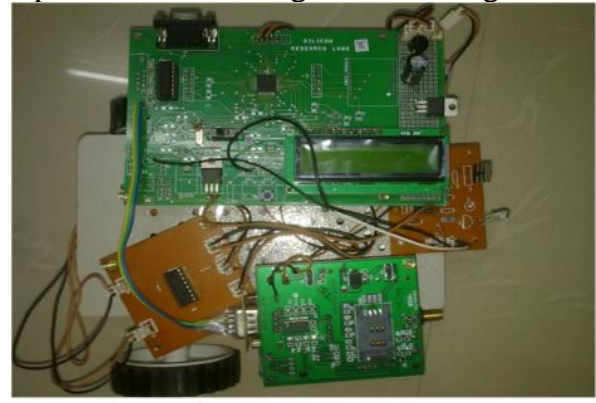

General objectives of the paper are defined as

a. To co-ordinate appliances and other devices through Short Message Service (SMS).

b. To effectively receive and transmit data via SMS 
c. To eliminate the need of being physically present in any location for tasks involving the operation of appliances within a household/office.

The robot is successfully controlled through the SMS. So that the area and range of the controlling any system is increases. Only limit is that there should be service network (coverage).

It is very simpler than other controlling robot system like Bluetooth, RF circuits. System controlling through voice needs extra circuit of voice recognizing kit. This kit wants extra power and also it becomes somewhat complex.

So use of SMS is very simple, easy, cheap and secure way of controlling the embedded system as compare to other controlling ways.

\section{Future Work}

Installing camera with GPRS system provide the live streaming of the places where a human can't reach. By adding GPS system, the exact position of the robot can be traced so the position of the robot can be known. In above both cases the acknowledge facility can have a tremendous scope.

\section{Conclusion}

The paper on "Controlling Robot through SMS with Acknowledging facility" has been successfully developed by integrating features of all the hardware components used. Low cost, easy to use for rural areas, automated operation, and Low Power consumption are the features of the robot. GSM as wireless data communication platform, the system is small, stable and reliable, with small delay, which can effectively overcome the past disadvantages of poor real-time and high operating costs existed in the system.

\section{Acknowledgment}

I would like to thank all teacher stop of Electronics and communication department of my college G.H.R.A.E.T. especially to Dr. S. L. Haridas and Prof. Sanjay Tembhurne for the helpful comments and preparing the integration subsection of this paper.

\section{References}

[1]. Nan Li, Shugen Ma," An Online Stair-Climbing Control Method for a Transformable Tracked Robot," Senior Member, IEEE , Bin Li, Minghui Wang, and Yuechao Wang, 2012.

[2]. Zhang Wen and Jiang Meng, "Design of Vehicle positioning System Based on ARM", 978-1-61284-109-0/11/@2011 IEEE

[3]. A R.Manshty, A.Rajabzadeh, Z.Forootan jahromi, " A Scenario-Based Mobile Application for Robot-assisted Smart Digital Homes" (IJCSIS) International Journal of Computer Science and Information Security, Vol. 8, No. 5, August 2010

[4]. Zhang Wen, "Research on embedded positioning system of vehicle, Manufacturing Automation" Vol 32, pp.152-154, July 2010.

[5]. Liu Lei, Jin Zhigang, Tao Chunhua, "Survey of virtualization technology research and realization, Geospatial information", Vol 1, pp.175-177, January 2009.

[6]. Xiang Shuaiqiu, Zhan Shaobin, Jiang Tao, On the main technological schemes and algorithm for "Vehicle location system, Geospatial information", Vol 6, pp.87-90, June 2008.

[7]. P. Ben-Tzvi, S. Ito, and A. A. Goldenberg, "Autonomous stair climbing with re-configurable tracked mobile robot," in Proc. IEEE Workshop Robot. Sens. Environ, 2007, pp. 1-6

[8]. R. Montúfar-Chaveznava, C. Paisano, J.M. Cañas, and E.D. Jódar, "Simulating telerobotics by cellular telephony", Proc. ICINCO, pp.329- 334, in 2005

[9]. D. López de Ipiña1, I. Vázquez2, J. Ruiz de Garibay3 and DavidSainz, "An architecture for sentient GPRS-enabled Microbots", IEEE CIRA, pp.145-150, 2005

[10]. Kubik T, "Use of cellular phone in mobile robot voice control" Proceedings of the 40th SICE Annual Conference. International Session Papers 25-27 July 2001.

[11]. Masuda R., "Multi sensor control system for rescue robot" IEEE/SICE/RSJ international conference on 08-11 Dec 1996. 